\title{
A Dempster-Shafer Theory based combination of classifiers for hand gesture recognition
}

\author{
Thomas Burger ${ }^{1,3}$, Oya Aran², Alexandra Urankar ${ }^{3}$, Alice Caplier ${ }^{1}$, Lale Akarun ${ }^{2}$ \\ ${ }^{1}$ Gipsa-Lab, Institut National Polytechnique de Grenoble, 46 av. Felix Viallet, Grenoble, \\ France \\ - \{firstname\}.\{lastname\}@ gipsa-lab.inpg.fr - \\ ${ }^{2}$ Dep. of Comp. Eng., Bogazici University, Bebek 34342, Istanbul, Turkey \\ - aranoya@boun.edu.tr, akarun@boun.edu.tr - \\ ${ }^{3}$ France Telecom R\&D employee during this research conduction
}

\begin{abstract}
As part of our work on hand gesture interpretation, we present our results on hand shape recognition. Our method is based on attribute extraction and multiple partial classifications. The novelty lies in the fashion the fusion of all the partial classification results are performed. This fusion is (1) more efficient in terms of information theory and leads to more accurate results, (2) general enough to allow heterogeneous sources of information to be taken into account: Each classifier output is transformed to a belief function, and all the corresponding functions are fused together with other external evidential sources of information.
\end{abstract}

Keywords: SVM, Expert systems, HMM, Belief functions, Hu invariants, Hand shape and gesture recognition, Cued Speech, probabilistic transform.

\section{Introduction}

Hand shape recognition is a widely studied topic which has a wide range of applications such as HCI [1], automatic gesture translators, tutoring tools for the hearing-impaired [2], [3], augmented reality, and medical image processing.

Even if this field is dominated by Bayesian methods, several recent studies deal with evidential methods, as they bring a certain advantage in the fashion uncertainty is processed in the decision making [4], [5].

The complete recognition of a hand shape with no constraint on the shape is an open issue. Hence, we focus on the following problem: (1) the hand is supposed to roughly remain in a plan which is parallel to the acquisition plan (2) only nine different shapes are taken into account (Fig. 1a). No assumptions are made on the respective location of the fingers (whether they are gathered or not, which drastically increases the inner variance of each shape); except for hand shapes 2 (gathered fingers) and 8 (as separated as possible), as this is their only difference. These nine hand shapes correspond to the gesture set used in Cued Speech [6]. 


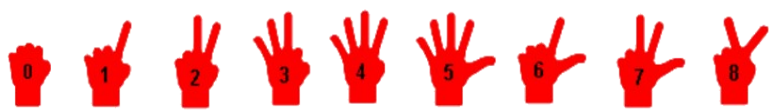

(a) Artificial representation of the hand shape classes.

\section{IIItydy}

(b) Segmented hand shape examples from real data

Fig. 1. The 9 hand shape classes

There are many methods already developed to deal with hand modeling and analysis. For a complete review, see [7], [8]. In this paper, we do not develop the segmentation aspect. Hence, we consider several corpora of binary images such as in Fig. 1b, as the basis of our work. The attribute extraction is presented in Section 2. The required classification tools are presented in Section 3. Section 4 is the core of this paper: we apply the decision making method, which is theoretically presented in [9], to our specific problem, and we use its formalism as a framework in which it is possible to combine classifiers of various nature (SVMs and expert systems) providing various partial information. Finally, Section 5 provides experimental results, and Section 6 discusses possible theoretical extensions.

\section{Attribute definition}

\subsection{Hu invariants}

The dimensionality of the definition space for the binary images we consider is very large, and it is intractable to use pixel values to perform the classification. One needs to find a more compact representation of the image. Several such binary image descriptors are to be found in the image compression literature [10]. They can be classified into two main categories:

- Region descriptors, which describe the binary mask of a shape, such as Zernike moments, Hu invariants, and grid descriptors.

- Edge descriptors, which describe the closed contour of the shape, such as Fourier descriptors, and Curvature Scale Space (CSS) descriptors.

Region descriptors are less sensitive to edge noise because of an inertial effect of the region description. Edge descriptors are more related to the way human compare shapes.

A good descriptor is supposed to obey several criteria, such as geometrical invariance, compactness, being hierarchical (so that the precision of the description can be truncated), and being representative of the shape.

We focus on $\mathrm{Hu}$ invariants, which are successful in representing hand shapes [11]. Note that parallel studies are conducted on promising Fourier-Mellin Descriptors. The $\mathrm{Hu}$ invariants' purpose is to express the mass repartition of the shape via several 
inertial moments of various orders, on which specific transforms ensure invariance to similarities.

Let us compute the classical definition of centered inertial moments of order $p+q$, for the shape (invariant to translation, as they are centered on the gravity center):

$$
m_{p q}=\iint_{x y}(x-\bar{x})^{p}(y-\bar{y})^{q} \delta(x, y) d x d y
$$

with $\bar{x}$ and $\bar{y}$ being the coordinates of the center of gravity of the shape and $\delta(x, y)=1$ if the pixel belongs to the hand shape and 0 otherwise. In order to make these moments invariant to scale, we normalize them:

$$
n_{p q}=\frac{m_{p q}}{m_{00}^{\frac{p+q}{2}+1}}
$$

Then, we compute the six Hu invariants, which are invariant to rotation, and mirror reflection:

$$
\begin{aligned}
S_{1}= & n_{20}+n_{02} \\
S_{2}= & \left(n_{20}+n_{02}\right)^{2}+4 \cdot n_{11}{ }^{2} \\
S_{3}= & \left(n_{30}-3 \cdot n_{12}\right)^{2}+\left(n_{03}-3 \cdot n_{21}\right)^{2} \\
S_{4}= & \left(n_{30}+n_{12}\right)^{2}+\left(n_{03}+n_{21}\right)^{2} \\
S_{5}= & \left(n_{30}-3 \cdot n_{12}\right) \cdot\left(n_{30}+n_{12}\right) \cdot\left(\left(n_{30}+n_{12}\right)^{2}-3 \cdot\left(n_{03}+n_{21}\right)^{2}\right) \\
& -\left(n_{03}-3 \cdot n_{21}\right) \cdot\left(n_{03}+n_{21}\right) \cdot\left(3 \cdot\left(n_{30}+n_{12}\right)^{2}-\left(n_{03}+n_{21}\right)^{2}\right) \\
S_{6}= & \left(n_{20}+n_{00}\right) \cdot\left(\left(n_{30}+n_{12}\right)^{2}-\left(n_{03}+n_{21}\right)^{2}\right) \\
& +4 \cdot n_{11}{ }^{2} \cdot\left(n_{30}+n_{12}\right) \cdot\left(n_{03}+n_{21}\right)
\end{aligned}
$$

A seventh invariant is available. Its sign permits to discriminate mirror images and thus, to suppress the reflection invariance:

$$
\begin{aligned}
S_{7}= & \left(3 \cdot n_{21}-n_{03}\right) \cdot\left(n_{30}+n_{12}\right) \cdot\left(\left(n_{30}+n_{12}\right)^{2}-3 \cdot\left(n_{03}+n_{21}\right)^{2}\right) \\
& -\left(n_{30}-3 \cdot n_{12}\right) \cdot\left(n_{03}+n_{21}\right) \cdot\left(3 \cdot\left(n_{30}+n_{12}\right)^{2}+\left(n_{03}+n_{21}\right)^{2}\right)
\end{aligned}
$$

The reflection invariance has been removed at the acquisition level and only left hands are processed. Hence, we do not need to discriminate mirror images. We nevertheless use $S_{7}$ as both the sign and the magnitude carry information: it sometimes occurs that hand shapes 3 and 7 (both with separated fingers) really look like mirror images. Finally, the attributes are: $\left\{S_{1}, S_{2}, S_{3}, S_{4}, S_{5}, S_{6}, S_{7}\right\}$. 


\subsection{Thumb Presence}

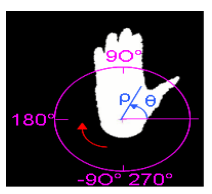

(a) Binary hand shape image.
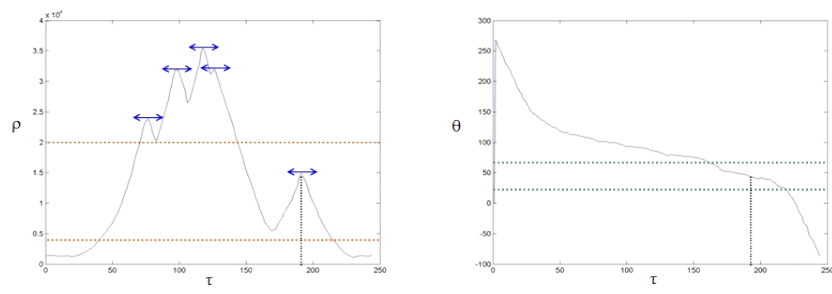

(b) Hand polar parametric representation along the curvilinear abscissa T (distance $\rho$ and angle $\theta$ ). The thumb is within a peculiar distance and angle range (horizontal lines).

Fig. 2. Thumb detection.

The thumb is an easy part to detect, due to its peculiar size and position with respect to the hand. Moreover, the thumb presence is a very discriminative piece of evidence as three hand shapes require the thumb and six do not require it. The thumb detector works as follows:

(1) Polar parametric definition: By following the contour of the hand shape, a parametric representation $\{\rho(T), \theta(T)\}$ is derived in polar coordinates (Fig.2a)

(2) Peak detection: After smoothing the parametric functions, (low-pass filtering and sub-sampling), the local maxima of the $\rho$ function are detected. Obviously, they correspond to the potential fingers (Fig.2b).

(3) Threshold adaptation: Thresholds must be defined on the distance and the angle values to indicate the region in which a thumb is plausible. The angle thresholds that describe this region are derived from morphological statistics [12] : in practice, the thumb angle with respect to the horizontal axis (Fig.2b) is between $20^{\circ}$ and $65^{\circ}$. The distance thresholds are derived from a basic training phase whose main purpose is to adapt the default approximate values (1/9 and 5/9 of the hand length) via a scale normalization operation with respect to the length of the thumb. Even if the operation is simple, it is mandatory to do so, as the ratio of the thumb length with respect to the total hand length varies from hand to hand.

(4) Peak measurement: If a finger is detected in the area defined by these thresholds, it is the thumb. Its height with respect to the previous local minima (Fig.2) is measured. It corresponds to the height between the top of the thumb and the bottom of the inter-space between the thumb and the index. This value is the thumb presence indicator (it is set to zero when no thumb is detected). In practice, the accuracy of the thumb detection (the thumb is detected when the corresponding indicator has a nonzero value) reaches $94 \%$ of true detection with $2 \%$ of false alarms.

The seven $\mathrm{Hu}$ invariants and the thumb presence indicator are used as attributes for the classification. 


\section{Classification tools}

\subsection{Belief functions and the Transfer Belief Model (TBM)}

In this section, we briefly present the necessary background on belief functions. For deeper comprehension of these theories, see [13] and [14].

Let $\Omega$ be the set of $N$ exclusive hypotheses $h_{1} \ldots h_{N}$. We call $\Omega$ the frame of discernment, or the frame, for short. Let $m$ (.) be a belief function on $2^{\Omega}$ (the powerset of $\Omega$ ) that represents our mass of belief in the propositions that correspond to the elements of $2^{\Omega}$ :

$$
\begin{aligned}
m: 2^{\Omega} & \rightarrow[0,1] \\
A & \mapsto m(A) \text { with } \sum_{A \subseteq \Omega} m(A)=1
\end{aligned}
$$

Note that:

-Belief can be assigned to non-singleton propositions, which allows modeling the hesitation between elements;

-In the TBM, it is possible to associate a belief in $\varnothing$. It corresponds to conflict in the model, throughout an assumption in an undefined hypothesis of the frame or throughout a contradiction between the information on which the decision is made.

To combine several belief functions (each associated to one specific captor) into a global belief function (under associativity and symmetry assumptions), one uses the conjunctive combination. For $N$ belief functions, $m_{1} \ldots m_{N}$, defined on the same frame $\Omega$, the conjunctive combination is defined as:

$$
\begin{aligned}
& (\cap): \overbrace{\mathfrak{B}^{\Omega} \times \mathfrak{B}^{\Omega} \times \ldots \times \mathfrak{B}^{\Omega}}^{N} \rightarrow \mathfrak{B}^{\Omega_{v}} \\
& m_{1}(n) m_{2}(n) \ldots(n) m_{N} \mapsto m_{(\cap)}
\end{aligned}
$$

with $\mathfrak{B}^{\Omega}$ being the set of belief functions defined on $\Omega$ and $m_{(\cap)}$ being the global combined belief function. Thus, $m_{(\cap)}$ is calculated as:

$$
m_{(\cap)}(A)=\sum_{A=A_{1} \cap . . \cap A_{N}}\left(\prod_{n=1}^{N} m_{n}\left(A_{n}\right)\right) \forall A \subseteq 2^{\Omega_{v}}
$$

The conjunctive combination means that, for each element of the power set, its belief is the combination of all the beliefs (from the $N$ sources) which imply it: it is the evidential generalization of the logical AND.

After having fused several beliefs, the knowledge in the problem is modeled via a function over $2^{\Omega}$, which expresses the potential hesitations in the choice of the solution. In order to provide a complete decision, one needs to eliminate this hesitation. For that purpose, we use the Pignistic Transform [14], which maps a belief function from $2^{\Omega}$ onto $\Omega$, on which a decision is easy to make. The Pignistic Transform is defined as:

$$
\operatorname{BetP}(h)=\frac{1}{1-m(D)} \sum_{h \in A, A \subset \Omega} \frac{m(A)}{|A|} \quad \forall h \in \Omega
$$


where $A$ is a subset of $\Omega$, or equivalently, an element of $2^{\Omega}$, and $|A|$ its cardinal when considered as a subset of $\Omega$. This transform corresponds to sharing the hesitation between the implied hypotheses, and normalizing the whole by the conflictive mass.

As an illustration of all these concepts, let us consider a simple example: Assume that we want to automatically determine the color of an object. The color of the object can be one of the primary colors: red $(R)$, green $(G)$ or blue $(B)$. The object is analyzed by two sensors of different kind, each giving an assumption of its color.

Table 1. Numerical example for belief function use

\begin{tabular}{|c|c|c|c|c|c|c|c|c|}
\hline & $Q$ & $\simeq$ & $\omega$ & ص & 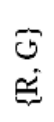 & ته & $\begin{array}{l}\text { जo } \\
\text { قै }\end{array}$ & تَ \\
\hline$m_{1}$ & 0 & 0.5 & 0 & 0 & 0.5 & 0 & 0 & 0 \\
\hline$m_{2}$ & 0 & 0 & 0 & 0 & 0 & 0 & 0.4 & 0.6 \\
\hline$m_{1}(\cap) m_{2}$ & 0.2 & 0.3 & 0.2 & 0 & 0.3 & 0 & 0 & 0 \\
\hline$B e t P$ & 0 & 0.56 & 0.44 & 0 & 0 & 0 & 0 & 0 \\
\hline
\end{tabular}

The observations of the sensors are expressed as belief functions $m_{1}($.$) and m_{2}($. and the frame is defined as $\Omega_{\text {color }}=\{\varnothing, \mathrm{R}, \mathrm{G}, \mathrm{B},\{\mathrm{R}, \mathrm{G}\},\{\mathrm{R}, \mathrm{B}\},\{\mathrm{B}, \mathrm{G}\},\{\mathrm{R}, \mathrm{G}, \mathrm{B}\}\}$ representing the hypothesis about the color of the object. Then, they are fused together into a new belief function via the conjunctive combination. As the object has a single color, the belief in union of colors is meaningless from a decision making point of view. Hence, one applies the Pignistic Transform on which a simple argmax decision is made. This is summarized and illustrated in Table 1.

\subsection{Support Vector Machines}

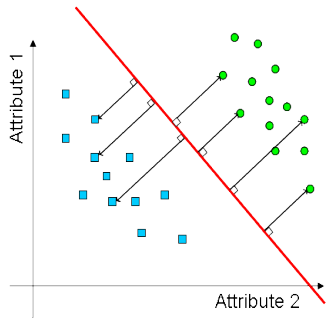

(a)

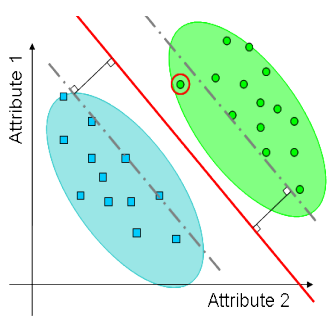

(b)

Fig. 3. (a) Combinatorial optimization of the hyperplane position under the constraints of the training corpus item positions. (b) The SVM provides good classification despite the bias of the training.

SVMs [15], [16] are powerful tools for binary classification. Their purpose is to extract the correlation of the attributes for each class by defining a separating hyperplane derived from a training corpus, which is supposed to be statistically 
representative of the classes involved. The hyperplane is chosen among all the possible hyperplanes through a combinatorial problem optimization, so that it maximizes the distance (called the margin) between each class and the hyperplane itself (Fig. 3a \& Fig. 3b).

To deal with the nine hand shapes in our database, a multiclass classification with SVMs must be performed. As SVMs are restricted to binary classification, several strategies are developed to adapt them for multiclass classification problems [17]. For that purpose, we have developed our own scheme [9], the Evidential Combination, which fuses the outputs of the SVMs using the belief formalism, and which provides a robust way of dealing with uncertainties. The method can be summarized by the following three steps:

(1) 36 SVMs are used to compare all the possible class pairs among nine classes;

(2) A belief function is associated to each SVM output, to model the partial knowledge brought by the corresponding partial binary classification;

(3) The belief functions are fused together with a conjunctive combination, in order to model the complete knowledge of the problem, and to make a decision according to its value.

Classically, SVM outputs are +1 or -1 , depending on the class chosen, but it remains a binary decision with respect of the two classes involved. Then, all the binary outputs are fused by a voting process. Unfortunately, , (1) the votes are sometimes in ties for two or more classes, (2) the various outputs are sometimes not consistent: SVM_1 chooses class_A rather than class_B, SVM_2 chooses class_B rather than class_C, and SVM_3, class_C rather than class_A. In order to deal with such situations, methods have been proposed to convert the SVM outputs into probabilistic distributions, but, in [17], these methods are said to be equivalent to voting. On the contrary, our Evidential Combination has proved to be efficient on various datasets [9].

\section{Decision scheme}

\subsection{Belief in the thumb presence}

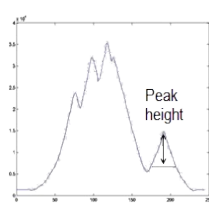

(a)

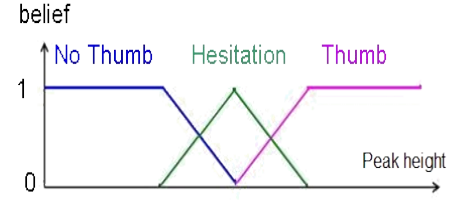

(b)

Fig. 4. (a) The peak height determines (b) the belief in the presence of the thumb

In order to fuse the information from the thumb presence indicator with the output of the SVM classifier, one needs to represent it with a belief function. As it is impossible to have a complete binary certitude on the presence of the thumb (it is possible to be misled at the thumb detection stage as explained previously), we use a belief function which authorizes hesitation in some cases. 
From an implementation point of view, we use a technique based on fuzzy sets, as explained in Fig. 4: The higher the peak of the thumb is, the more confident we are in the thumb presence. This process is fully supported by the theoretical framework of belief functions, as the set of the finite fuzzy sets defined on $\Omega$ is a subset of $\mathfrak{B}^{\Omega}$ (the set of belief functions defined on $\Omega$ ). Moreover, as belief functions, fuzzy sets have special properties which make them convenient to fuse with the conjunctive combination [18]. Note that it is roughly the same technique as the one used to associate a belief function to the output of each SVM.

The three values that define the support of the hesitation in Fig. $4 \mathrm{~b}$ have been manually fitted according to observations on the training set. Making use of the "fuzziness" of the threshold between the thumb presence and absence, these values are not necessarily precisely settled. In practice, they are defined via three ratios $(1 / 5$, $1 / 20$ and 1/100) of the distance between the center of palm and the furthest element from it of the contour.

Then, the belief in the presence of the thumb can be associated to a belief in some hand shapes to produce a partial classification: In hand shapes $0,1,2,3,4$, and 8 , there is no thumb, whereas it is visible for shapes 5, 6 and 7. In case of hesitation in the thumb presence, no information is brought and the belief is associated to $\Omega$.

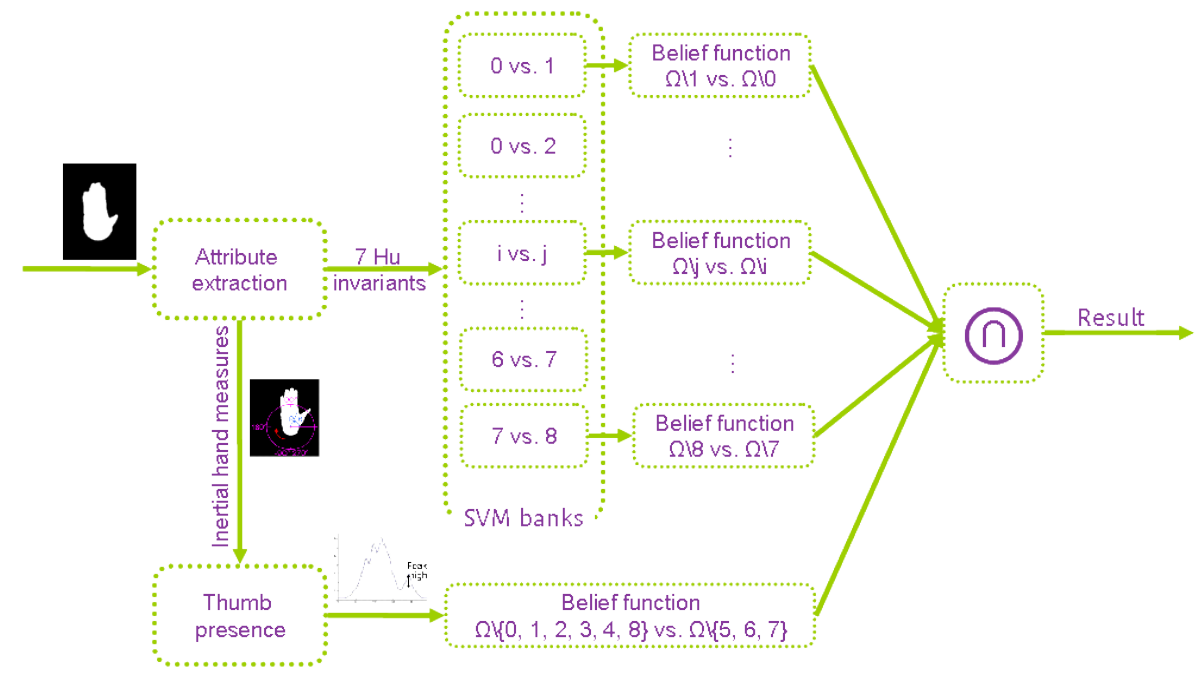

Fig. 5. Global fusion scheme for hand shape classification

\subsection{Partial classification fusion}

Thanks to the evidential formalism, it is possible to fuse partial information from various classifiers (36 SVMs and 1 expert system) through the conjunctive combination (Fig. 5). In that fashion, it is possible to consider a SVM-based system and integrate it into a wider data fusion system. 
This fusion provides a belief function over the powerset $2^{\Omega}$ of all the possible hand shapes $\Omega$. This belief is mapped over $\Omega$ via the Pignistic Transform, to express our belief in each singleton element of $\Omega$. Then, the decision is made by an argmax function over $\Omega$.

$$
D^{*}=\underset{\Omega}{\operatorname{argmax}}(\operatorname{BetP}(.))
$$

\section{Results}

In this section, we present various results on the methodology described above. In 5.1, the database and the evaluation methods are detailed. In 5.2, the experiments and corresponding results are given.

\subsection{Database \& Methodology}

The hand shape database used in this work is obtained from Cued Speech videos. The transition shapes are eliminated manually and the remaining shapes are labeled and used in the database as binary images representing the 9 hand shapes (Fig. 1).

Table 2. Details of the database

\begin{tabular}{ccc}
\hline \hline Hand Shape & $\begin{array}{c}\text { Corpus 1 } \\
\text { (Training set) }\end{array}$ & $\begin{array}{c}\text { Corpus 2 } \\
\text { (Test set) }\end{array}$ \\
\hline 0 & 37 & 12 \\
1 & 94 & 47 \\
2 & 64 & 27 \\
3 & 84 & 36 \\
4 & 72 & 34 \\
5 & 193 & 59 \\
6 & 80 & 46 \\
7 & 20 & 7 \\
8 & 35 & 23 \\
\hline Total & 679 & 291 \\
\hline \hline
\end{tabular}

The training and test sets of the database are formed such that there is no strict correlation between them. To ensure this, two different corpuses are used in which a single user is performing two completely different sentences using Cued Speech. The respective distribution of the two corpora are given in Table 2. The statistical distribution of the hand shapes is not balanced at all within each corpus. The reason of such a distribution is related to the linguistics of Cued Speech, and is beyond our scope.

For all the experiments, Corpus 1 is used as the training set for the SVMs and Corpus 2 is used as the test set. Since the real labels are known, we use the classical definition of the accuracy to evaluate the performance of the classifier: 


$$
\text { Accuracy }=100 \cdot \frac{\text { Number Of Well Classified Iten }}{\text { Total Number Of Items }}
$$

To fairly quantify the performances of each classification procedure, two indicators are used: (1) The difference between the respective accuracies, expressed in the number of point $\triangle$ Point, and (2) the percentage of avoided mistake $\% A v M i s$ :

$$
\begin{aligned}
& \Delta \text { Point }=\text { Accuracy }(\text { Method_2 })-\operatorname{Accuracy}\left(\operatorname{Method} \_1\right) \\
& \% A v M i s=100 \cdot \frac{\text { Number of Avoided Mistakes }}{\text { Total Number of Mistakes }} \\
& =100 \cdot \frac{\Delta \text { Point }}{100-\text { Accuracy }\left(\text { Method_1 }_{1}\right)}
\end{aligned}
$$

\subsection{Experiments}

The goal of the first experiment is to evaluate the advantage of the evidential fusion for the SVM. Thus, we compare the classical methods for SVM multi-classification to the one of [9]. For both of the methods, the training is the same and the SVMs are tuned with respect to the training set and the thumb information is not considered.

For the implementation of the SVM functionalities, we use the open source C++ library LIBSVM [19]. We use:

- C-SVM, which is an algorithm to solve the combinatorial optimization. The cost parameter is set to 100,000 and termination criteria to 0.001 .

- Sigmoid kernels in order to transform the attribute space so that it is linearly separable:

$$
\begin{aligned}
& \operatorname{Ker}_{\gamma, R}(u, v)=\tanh \left(\gamma \cdot u^{T} \cdot v+R\right) \\
& \text { with } \gamma=0.001 \text { and } R=-0.25
\end{aligned}
$$

For the evidential method, we have made various modifications on the software so that the SVM output is automatically presented throughout the evidential formalism [9].

The results are presented in Table 3 , as the test accuracy of the classical voting procedure and the default tuning of the evidential method. The improvement in $\triangle$ Point is worth 1.03 points and corresponds to an avoidance of mistakes of $\%$ AvMis $=11.11 \%$.

The goal of the second experiment is to evaluate the advantage of the thumb information. For that purpose, we add the thumb information to the evidential method. Thus, the training set is used to set the two thresholds, which defines the possible distance with respect to the center of palm. However, the thumb information is not used during the training of the SVMs as they only work on the Hu invariants, as explained in Fig. 5. The results with and without the thumb indicator are presented in Table 3. 
Table 3. Results for experiments $1 \& 2$

\begin{tabular}{c|ccc}
\hline & $\begin{array}{c}\text { Classical } \\
\text { Voting } \\
\text { procedur } \\
e\end{array}$ & $\begin{array}{c}\text { Evidential method } \\
\text { Default } \\
\text { (no thumb } \\
\text { detection) }\end{array}$ & $\begin{array}{c}\text { With Thumb } \\
\text { Detection }\end{array}$ \\
\hline $\begin{array}{c}\text { Test } \\
\text { Accuracy }\end{array}$ & $90.7 \%$ & $91.8 \%$ & $92.8 \%$ \\
\hline \hline
\end{tabular}

The evidential method that uses the thumb information provides an improvement of 2.06 points with respect to the classical voting procedure, which corresponds to an avoidance of $22.22 \%$ of the mistakes. Table 4 presents the corresponding confusion matrix for the test set: Hand shape 3 is often misclassified into other hand shapes, whereas, on the contrary, hand shape 1 and 7 gather a bit more misclassification from other hand shapes. Moreover, there are only three mistakes between THUMB and NO_THUMB super-classes.

Table 4. Confusion matrix for the second method on Corpus 2, with the Thumb and NoThumb superclasses framed together.

\begin{tabular}{|c|c|c|c|c|c|c|c|c|c|}
\hline & 0 & 1 & 2 & 3 & 4 & 5 & 6 & 7 & 8 \\
\hline 0 & 12 & 0 & 0 & 0 & 0 & 0 & 0 & 0 & 0 \\
\hline 1 & 0 & 46 & 0 & 0 & 0 & 0 & 0 & 0 & 1 \\
\hline 2 & 0 & 2 & 23 & 2 & 0 & 0 & 0 & 0 & 0 \\
\hline 3 & 0 & 2 & 0 & 29 & 2 & 2 & 1 & 0 & 0 \\
\hline 4 & 0 & 0 & 0 & 1 & 32 & 0 & 0 & 0 & 1 \\
\hline 5 & 0 & 0 & 0 & 0 & 0 & 58 & 0 & 1 & 0 \\
\hline 6 & 0 & 0 & 2 & 0 & 0 & 0 & 41 & 3 & 0 \\
\hline 7 & 0 & 0 & 0 & 0 & 0 & 0 & 1 & 6 & 0 \\
\hline 8 & 0 & 0 & 0 & 0 & 0 & 0 & 0 & 0 & 23 \\
\hline
\end{tabular}

\section{Discussion and theoretical outlook}

We have presented a method and experimental data which demonstrates that the Evidential Combination of SVM is an interesting tool to fuse SVM-processed data in a wider data fusion scheme. Consequently, the next challenge is to find a method so that other non-evidential classifier outputs can be considered in the evidential framework. In fact, it is required to guarantee that this method is available on SVM and expert systems, but also on other classifiers, in what we have called a "wider fusion scheme".

Of course, as explained in [9], the method also fits any binary classifier in which the minimum distance to the separating hyperplane is known (the margin), as it is the only required point to define the belief function. In case no such margins are available, it is possible to experimentally define them, via training or cross validation. 
In [20], we have applied this paradigm to video recognition of American Sign Language. Each sign is modeled by a Hidden Markov Model (HMM) and binary classifiers are derived from pairwise comparisons of the HMM likelihood scores. The very satisfying results show the efficiency of the method. But, it also demonstrates that it is not limited to binary classifiers; as a matter of fact, the derivation of binary classifiers from pairwise comparison is somehow artificial, and as a consequence, it is more straightforward to directly handle such unary classifiers (such as HMM). Hence, our method is also efficient on unary classifiers based on their generative properties.

Moreover, let us point out another fact: Practically, the result of a set of HMMs is a set of likelihood scores, i.e. a subjective probability distribution prior to any normalization. Thus, this work is a good source of inspiration to provide a method to convert a probability into a belief function. As several such transformation methods are defined in incompatible fashions in the literature, such as [21], and as the relationships between belief functions and probabilities are a topic of strong debates [22], this subject should be carefully considered. Thus, we do not address it with respect to its epistemological dimension, and we stick to strict and simple computational considerations. As an introduction to further theoretical development, we propose to consider the following computation as an eventual interesting way to convert a probability distribution into a belief function: First of all, we make the assumption that the Pignistic Transform defines the relationships between probabilities and BF (which is a strong assumption on which the entire community does not agree). Moreover, we consider in this computational outlook that, any difference in the probability of two possible outcome gives an evidential clues that the outcome of higher probability is more likely to be believed in; and the quantification of the corresponding belief function is proportional to the difference of the probabilities (this later assumption is slightly more likely to be taken for granted). It is possible to derive $N-1$ belief functions from the comparison of $N$ ordered probability (from the highest value to the smallest) values corresponding to $N$ outcomes. The Pignistic Transform of their conjunctive combination should give the original probability distribution:

$$
\operatorname{PigT}\left(m_{1(n)} m_{2,(n)} \ldots \text { (n) } m_{N-1}\right)=\mathrm{p}
$$

with PigT corresponding to the pignistic transform. As, any of the $m_{i}$ belief functions is defined on the powerset of two outcomes, it is possible to simplify the computation of the conjunctive combination (where $m_{(\wedge)}$ corresponds to the results of the combination of the $m_{i}$ ) :

$$
\left\{\begin{array}{l}
m_{(\cap)}\left(\mathcal{C}^{1}\right)=m_{1}\left(\mathcal{C}^{1}\right) \\
m_{(\cap)}\left(\left\{\mathcal{C}^{1}, \mathcal{C}^{2}\right\}\right)=m_{2}\left(\left\{\mathcal{C}^{1}, \mathcal{C}^{2}\right\}\right) \cdot m_{1}(\Omega) \\
\vdots \\
m_{(\Omega)}\left(\left\{\mathcal{C}^{1}, \ldots, \mathcal{C}^{i}\right\}\right)=m_{i}\left(\left\{\mathcal{C}^{1}, \ldots, \mathcal{C}^{i}\right\}\right) \cdot \prod_{j=1}^{i-1} m_{j}(\Omega) \\
\vdots \\
m_{(\cap)}(\Omega)=\prod_{j=1}^{N-1} m_{j}(\Omega) \\
m_{(\Omega)}(A)=0 \quad \forall \text { other } A \in 2^{\Omega}
\end{array}\right.
$$


Then, the application of the pignistic transform gives:

$$
\left\{\begin{array}{lr}
\operatorname{BetP}\left(\mathcal{C}^{1}\right)=m_{(ก)}\left(\mathcal{C}^{1}\right)+\frac{m_{(ก)}\left(\left\{\mathcal{C}^{1}, \mathcal{C}^{2}\right\}\right)}{2}+\cdots+\frac{m_{(ก)}\left(\left\{\mathcal{C}^{1}, \ldots, \mathcal{C}^{i}\right\}\right)}{i}+\cdots+\frac{m_{(ก)}(\Omega)}{N} \\
\operatorname{BetP}\left(\mathcal{C}^{2}\right)= & \frac{m_{(ก)}\left(\left\{\mathcal{C}^{1}, \mathcal{C}^{2}\right\}\right)}{2}+\cdots+\frac{m_{(ก)}\left(\left\{\mathcal{C}^{1}, \ldots, \mathcal{C}^{i}\right\}\right)}{i}+\cdots+\frac{m_{(ก)}(\Omega)}{N} \\
\vdots & \frac{m_{(ก)}\left(\left\{\mathcal{C}^{1}, \ldots, \mathcal{C}^{i}\right\}\right)}{i}+\cdots+\frac{m_{(ก)}(\Omega)}{N} \\
\operatorname{BetP}\left(\mathcal{C}^{i}\right)= & \frac{m_{(ก)}(\Omega)}{N} \\
\vdots &
\end{array}\right.
$$

where $C^{i}$ corresponds to the $i^{\text {th }}$ outcome. As we have assumed that $\operatorname{BetP}\left(C^{i}\right)=\mathrm{P}\left(C^{i}\right)$, one has :

$$
m_{i}(\Omega)=1-i \cdot \frac{\mathrm{P}\left(\mathcal{C}^{i}\right)-\mathrm{P}\left(\mathcal{C}^{i+1}\right)}{\prod_{k=1}^{i-1} m_{k}(\Omega)}
$$

which can be iteratively computed, and which set the $N-1 m_{i}$ with respect to the $N-1$ pairwise subtractions $\mathrm{P}\left(C^{i}\right)-\mathrm{P}\left(C^{i+1}\right)$ of the probability distribution.

\section{Conclusion}

In this paper, we propose to apply a belief-based method for SVM fusion to hand shape recognition. Moreover, we integrate it in a wider classification scheme which allows taking into account other sources of information, by expressing them in the Belief Theories formalism. The results are better than with the classical methods (more than 1/5 of the mistakes are avoided) and the absolute accuracy is high with respect to the number of classes involved. Directions for future work are presented in the last section, where we derive from a computational point of view, a method that could potentially convert a probability distribution into a belief function. The consequences of this computation from an information theory point of view are not explored yet, so that it is not possible to assess the interest of this work yet, but this is the matter of our next investigations.

Acknowledgments. This work is the result of a cooperation supported by SIMILAR, European Network of Excellence (www.similar.cc). It has partially been supported and financed by France Telecom R\&D.

\section{References}

1. Pavlovic, V., Sharma, R. and Huang, T. S., 1997. Visual Interpretation of Hand Gestures for Human-Computer Interaction: A Review, IEEE Transactions on Pattern Analysis and Machine Intelligence, vol. 19, n. 7, p. 677-695. 
2. Ong, S.C.W. and Ranganath, S., 2005. Automatic Sign Language Analysis: A Survey and the Future beyond Lexical Meaning, IEEE Transactions on Pattern Analysis and Machine Intelligence, vol. 27, pp. 873-891.

3. Aran, O., Ari, I., Benoit, A., Campr, P., Carrillo, A.H., Fanard, F., Akarun, L., Caplier, A. and Sankur, B., 2008. SignTutor: An Interactive System for Sign Language Tutoring, IEEE Multimedia, accepted for publication.

4. Quost, B., Denoeux, T., and Masson, M.-H., 2007. Pairwise classifier combination using belief functions. Pattern Recognition Letters, Vol. 28, Issue 5 , pp. 644-653.

5. Capelle, A.-S., Colot, O., Fernandez-Maloigne, C., 2004. Evidential segmentation scheme of multi-echo MR images for the detection of brain tumors using neighborhood information. Information Fusion, Volume 5, Number 3, pp. 203-216.

6. Cornett, R. O., 1967. Cued Speech, American Annals of the Deaf.

7. Derpanis, K. G., 2004. A review on vision-based hand gestures, internal report.

8. Wu, Y., Huang, T.S., 2001. Hand modeling, analysis, and recognition for vision based Human-Computer Interaction, IEEE Signal Processing Magazine, vol. 21, pp.51-60.

9. Burger , T., Aran, O., and Caplier, A., 2006. Modeling hesitation and conflict: A beliefbased approach, In Proc. ICMLA.

10. Caplier, A., Bonnaud, L., Malassiotis, S., and Strintzis, M., 2004. Comparison of 2D and 3D analysis for automated Cued Speech gesture recognition, In proc. SPECOM, St Petersburg, Russia.

11. Zhang, D., and Lu, G., 2003. Evaluation of MPEG-7 shape descriptors against other shape descriptors, Multimedia Systems, vol. 9, issue 1.

12. Norkin, C.C., and Levangie , P.K., 1992. Joint structure and function. (2nd ed.). Philadelphia: F.A. Davis.

13. Shafer, G., 1976. A Mathematical Theory of Evidence, Princeton University Press.

14. Smets, P., and Kennes, R., 1994. The transferable belief model. Artificial Intelligence, Vol. 66(2), pp. 191-234.

15. Boser, B., Guyon, I., and Vapnik, V., 1995. A training algorithm for optimal margin classifiers, In Proc. Fifth Annual Workshop on Computational Learning Theory.

16. Cortes, C., and Vapnik, V., 1995. Support-vector network, Machine Learning, Vol. 20, pp. 273-297.

17. Hsu, C.-W., and Lin, C.-J., 2002. A comparison of methods for multi-class support vector machines, IEEE Transactions on Neural Networks, Vol. 13, pp. 415-425.

18. Denoeux, T., 2000. Modeling vague beliefs using fuzzy-valued belief structures. Fuzzy Sets and Systems, Vol. 116(2), pp. 167-199.

19. Chang, C.-C., and Lin, C.-J., 2001. LIBSVM: a library for support vector machines. Software available at http://www.csie.ntu.edu.tw/ cjlin/libsvm

20. Aran, O., Burger, T., Caplier A., Akarun, L., 2008. A Belief-Based Sequential Fusion Approach for Fusing Manual and Non-Manual Signs. Pattern Recognition, accepted for publication.Sudano, J.J., 2002. Inverse pignistic probability transforms. In Proc. Information Fusion.

21. Cobb, B., Shenoy P., 2006. On the plausibility transformation method for translating belief function models to probability models. Int. J. Approx. Reasoning, Vol. 41(3), pp. 314-330. 